\title{
Polyaniline-based Electrodes: Recent Application in Supercapacitors and Next Generation Rechargeable Batteries
}

\author{
Silas K. Simotwo and Vibha Kalra \\ Department of Chemical and Biological Engineering, Drexel University, 3141 Chestnut Street, \\ Philadelphia, PA 19104, USA. \\ Corresponding author: Email: vk99@drexel.edu
}

This review highlights the versatility of polyaniline (PANI) for application in the electrodes of energy storage devices - supercapacitors and next-generation batteries (sodium-ion - SIB and lithium-sulfur - LiS). Supercapacitor electrodes with high energy storage capability and cycle life have been developed based on both neat PANI as well as its composites with carbon or inorganic pseudocapacitors. In batteries, tailorable synthesis of PANI to confine/coat nanostructures of electroactive materials $\left(\mathrm{Na}^{+}\right.$host materials in SIBs or sulfur in LiS) has resulted in notable improvement in utilization, stability and durability of these materials due to PANI's high conductivity, specific material interaction and its ability to ameliorate stress on the nanostructures caused by large volume changes during cycling. Limitations of PANI in such electrodes are discussed and possible future directions have been proposed.

\section{Introduction}

Quest for green energy, depleting energy reserves and the ever increasing energy demand for applications such as portable electronics, electrified transportation and grid-level energy storage have accelerated research in sustainable energy alternatives.[1] For the last two decades, lithium ion batteries (LIBs) have transformed the portable electronics industry owing to their high energy density and long life cycle. However, new market demand for sustainable higher energy and power sources calls for the development of new generation of LIBs or alternative energy storage devices. Rechargeable batteries such as lithium-air, lithium-sulfur (LiS), sodium-ion (SIB), and supercapacitors have received intense interest recently as energy storage prospects. The appeal for these energy devices lies in the natural abundance of their electrode materials, benign environmental impact, well-understood electrochemistry and promising energy storage capability.

Although important developments have been made on electrodes for supercapacitors and rechargeable batteries in recent literature, significant challenges related to electrochemical performance/utilization and durability remain.[2,3] The low electrochemical utilization is attributed to electrode morphology (small accessible surface area) and inadequate conductivity whereas the poor durability is related to electrode structural instability and/or loss of active materials during cycling. Therefore, there has been plenty of research focused on studying new electrode materials, nanocomposites and architectures in an attempt to address the aforementioned performance deficiencies. Polyaniline (PANI) is a readily available p-type conducting polymer which has commanded considerable attention for application in electrodes of most energy devices either as a sole electrode active material or as a supplement material to 
enhance performance. PANI is commonly synthesized through chemical oxidative polymerization of aniline using a strong oxidant. Electrochemical synthesis of polyaniline has also been widely applied. PANI can exist in three different oxidation states: leucoemaraldine, emaraldine base is the partially-oxidized and pernigraniline which are the fully reduced, partially oxidized and fully oxidized states, respectively.[4] Synthesis of PANI is largely carried out in acidic conditions, which result in a protonated/doped emeraldine salt. External doping or dedoping of the emeraldine salt has also been achieved using an acid or base, respectively.[5] Conductivity of PANI is strongly dependent on the degree of oxidation and the doping. Doped emeraldine base is the most conductive state, with conductivity ranging from 0.1 to over 100 S/cm.[6] Un-doped emaraldine and the fully reduced/oxidized states show conductivity in the order of $10^{-10} \mathrm{~S} / \mathrm{cm}$. PANI nanostructures are prepared either via a template-assisted method where an external soluble or insoluble substrate with pre-formed nanoscale features such as a metal oxide is employed or template-free technique where material's ability to self-assemble into various morphologies depending on the synthesis conditions $(\mathrm{pH}$, temperature, nature of dopants, etc.), is exploited. [7] [8].

This short review will provide a synopsis of recent research in the application of PANI and its nanocomposites in electrodes for supercapacitors and new generation rechargeable batteries. Focus will be placed on highlighting how PANI properties such as easy and flexible synthesis process, unique chemistry/redox process, mechanical flexibility and high electrical conductivity contribute to the high, reversible capacity and long term stability of electrodes for supercapacitors, SIBs and LiS batteries. We will also discuss the limitations of PANI application in such electrodes and possible future directions will be proposed.

\section{PANI in supercapacitors}

PANI is the most studied member of the conducting polymer family for supercapacitor application due to its excellent electrical conductivity, high theoretical pseudocapacitance (750 F $\mathrm{g}^{-1}$ )[9] derived from its reversible redox reactions and easy synthesis. However, although its application in supercapacitor electrodes has shown tremendous promise thus far, some lingering shortcomings prevent the realization of its optimal performance. These limitations include: (a) low capacitance retention at high charge/discharge rates and (b) poor durability after long term cycling (usually after $\sim 1000$ cycles).[10-12] The latter is caused by structural deterioration of PANI due to large volume changes associated with repeated doping and de-doping and overoxidation of the polymer at high potential.[13] The former limitation is caused by the morphology, which affects electrolyte penetration and hence ion transfer, and electrical conductivity of the PANI electrodes. This is in addition to the transport limitations at the polymer-electrolyte and electrode-current collector interfaces. In this section, we will examine the research effort within the last three years to address these PANI shortcomings. These efforts have included improvement of PANI electrode architectures, development of PANI composites and adoption of redox active electrolytes.

PANI-based Composites as Supercapacitor Cathodes 
Bulk of the PANI supercapacitor research so far has focused on nanocomposites of PANI with carbon and/or inorganic compounds to reinforce its electrical conductivity and/or mechanical stability to improve rate capability and cycle life performance. PANI/carbon composites have particularly been extensively studied since carbon is a readily available material with excellent electrical conductivity and mechanical properties.[14] A synergistic combination of excellent electrical conductivity, high surface area/pore volume and mechanical resilience of carbonaceous backbone and high pseudocapacitance of PANI is realized in such hybrid electrodes.[15] Of the carbon family, graphene has attracted notable attention as a viable host/substrate material for PANI. Graphene possesses large theoretical surface area $\left(\sim 2600 \mathrm{~m}^{2} / \mathrm{g}\right)$, superior chemical/mechanical stability, good processability and potential for scalability. Moreover, graphene is considered one of the best candidates for flexible electrode materials. [16] The resultant graphene/PANI nanocomposite electrodes have demonstrated high capacitance, long cycle life and impressive flexibility in supercapacitors. [17] The synthesis and application of graphene/PANI nanocomposites, has been comprehensively reviewed recently and the reader is directed to these reviews for detailed information.[18,19] Recently, our lab developed binderfree freestanding PANI-coated porous carbon nanofiber electrodes which achieved SC of $409 \mathrm{~F}$ $\mathrm{g}^{-1}$ at $0.1 \mathrm{~A} \mathrm{~g} \mathrm{~g}^{-1,} 80 \%$ of which was retained at a high current density of $10 \mathrm{~A} \mathrm{~g}^{-1}$.[20] Control experiments using PANI-coated, non-porous carbon nanofibers delivered much lower SC at the same current densities indicating that the double layer capacitance of the underlying carbon was retained and true integration of double layer and pseudocapacitance was achieved. Overall, the PANI/carbon composite electrodes have demonstrated good initial specific capacitance (SC) and reasonable capacitance retention (>80 \%) after long cycling (1000-5000 cycles) as illustrated in table 1.[21-23]. Fabrication and application of PANI/carbon electrodes, however, often face challenges such as complex procedure for preparation of hierarchical carbons, low loading of deposited PANI and low capacitance contribution of the carbon backbone (if any).[24,25]

Composites of PANI with transition metal oxides have also been studied owing to the inherently high pseudocapacitance of these materials.[26] $\mathrm{MnO}_{2}$ is considered as one of the promising members of the metal oxide group for supercapacitor applications due to its low cost and ecofriendliness in addition to excellent theoretical pseudocapacitance (1370 $\left.\mathrm{F} \mathrm{g}^{-1}\right)$.[27] Its composite with PANI has shown promising electroactivity where PANI acts not only as nanostructured scaffold for $\mathrm{MnO}_{2}$ but also as a conductive medium. Composites of PANI with other transition metal oxides such as nanostructured $\mathrm{V}_{2} \mathrm{O}_{5}, \mathrm{TiO}_{2}, \mathrm{RuO}_{2}$ and hollow $\mathrm{MoO}_{3}\left(\mathrm{~h}-\mathrm{MoO}_{3}\right.$ ) have also been investigated with good reported electrochemical performance.[28-30] Unfortunately, high cost, low conductivity and environmental unfriendliness of some of these oxides hinder the development of their composites with PANI.

\section{Pure PANI Cathodes}

Supercapacitor cathodes based on neat PANI (without use of additives) can potentially provide high electrode-level specific energy density. To offset PANI's mechanical instability and to maximize its utilization, interesting PANI architectures based on 1-D and 3-D interconnected nanostructures have been developed and studied for supercapacitors applications.[31,32] The nanostructured morphology is imperative since most of the PANI redox reactions occur at or 
near the electrode/electrolyte interface. The nanostructures help to truncate charge transfer pathways, relief strains within the electrode due to electroactivity and in some cases, enable new reactions not possible within bulk materials. [33] Synthesis techniques and application of various 1-D PANI nanomaterials have been studied for several years now. Recently hollow nanofibers synthesized via template technique [10] and nanotubes prepared via template-less process (selfassembly) [34] delivered high SCs of $601 \mathrm{~F} \mathrm{~g}^{-1}$ and $625 \mathrm{~F} \mathrm{~g}^{-1}$, respectively. The excellence in electroactivity of these 1-D nanostructures emanate from their morphology marked by high aspect ratio, porous surface and/or tubular morphology, which enables good electrode/electrolyte interaction, leading to efficient charge transfer to the reaction interface. Unfortunately, most of the supercapacitor electrodes based on 1-D PANI nanostructures still display undesirable loss in capacitance as shown in table 1 below. In comparison, recent works based on porous 3-D interconnected PANI nanostructures have shown promising capacitance retention capability at both higher current densities and after over 1000 cycles. Such improvement stems from the 3-D intertwined nanostructured network, which enables continuous electron conductivity as well as enhanced mechanical stability, and the hierarchical porosity, which facilitates faster ion diffusion/higher rate capability. Moreover, the macropores within such 3-D structures possibly relieve the stresses associated with volume changes during the doping/de-doping of PANI leading to enhanced cycling stability. Recently novel synthetic method was adopted where dopants acts as crosslinkers to synthesis conducting polymer gels (CPGs).[35] Crosslinked 3-D PANI hydrogel fabricated using phytic acid as both a gelator and a dopant showed excellent electron conductivity of $0.11 \mathrm{~S} / \mathrm{cm}$ and high capacitance retention of $93 \%$ at $5 \mathrm{~A} \mathrm{~g}^{-1}$ with $83 \%$ capacitance retention after 10000 cycles.[36] Similarly, a robust 3-D crosslinked PANI (see figure 2) electrode developed via concurrent inorganic ( $\mathrm{HCl}$ ) and organic acid (Phytic acid, PA) doping of PANI during the self-assembly process delivered a SC of $350 \mathrm{~F} \mathrm{~g}^{-1}$ which was found to be stable over a current density range of 1 to $40 \mathrm{~A} \mathrm{~g}^{-1}$.[37]

\section{Redox-active Electrolytes for PANI Supercapacitors}

An alternative route for the enhancement of PANI-based electrode pseudocapacitance and durability has been shown recently via modification of the conventional electrolytes using redox active agents such as hydroquinone.[38] The redox active species provide additional Faradaic reactions and can increase electrolyte conductivity and decrease charge transfer resistance leading to improved pseudocapacitance and durability. Vonlanthen and co-workers designed a supercapacitor consisting of symmetric PANI electrodes and $1 \mathrm{M} \mathrm{H}_{2} \mathrm{SO}_{4}$ electrolyte modified with benzoquinone-hydroquinone (HQ-BQ) mediators.[39] A SC of $524 \mathrm{~F} \mathrm{~g}^{-1}$ and unprecedented capacitance retention over 50000 cycles was reported. In comparison, PANI tested with no redox species showed a SC of $418 \mathrm{~F} \mathrm{~g}^{-1}$ and a $20 \%$ loss in capacitance after 2800 cycles.

Table 1. Polyaniline-based supercapacitors - Fabrication techniques, electroactivity (per gram of total electrode) at low and high current densities (CD), and cycle life.

\begin{tabular}{llll}
\hline & Low CD rate & High CD rate & \% Retention \\
SC in F g & SC in F g & (\# cycles) \\
& (Current Density) & (Current Density) & \\
\hline
\end{tabular}

PANI hybrids

PANI wt \% 


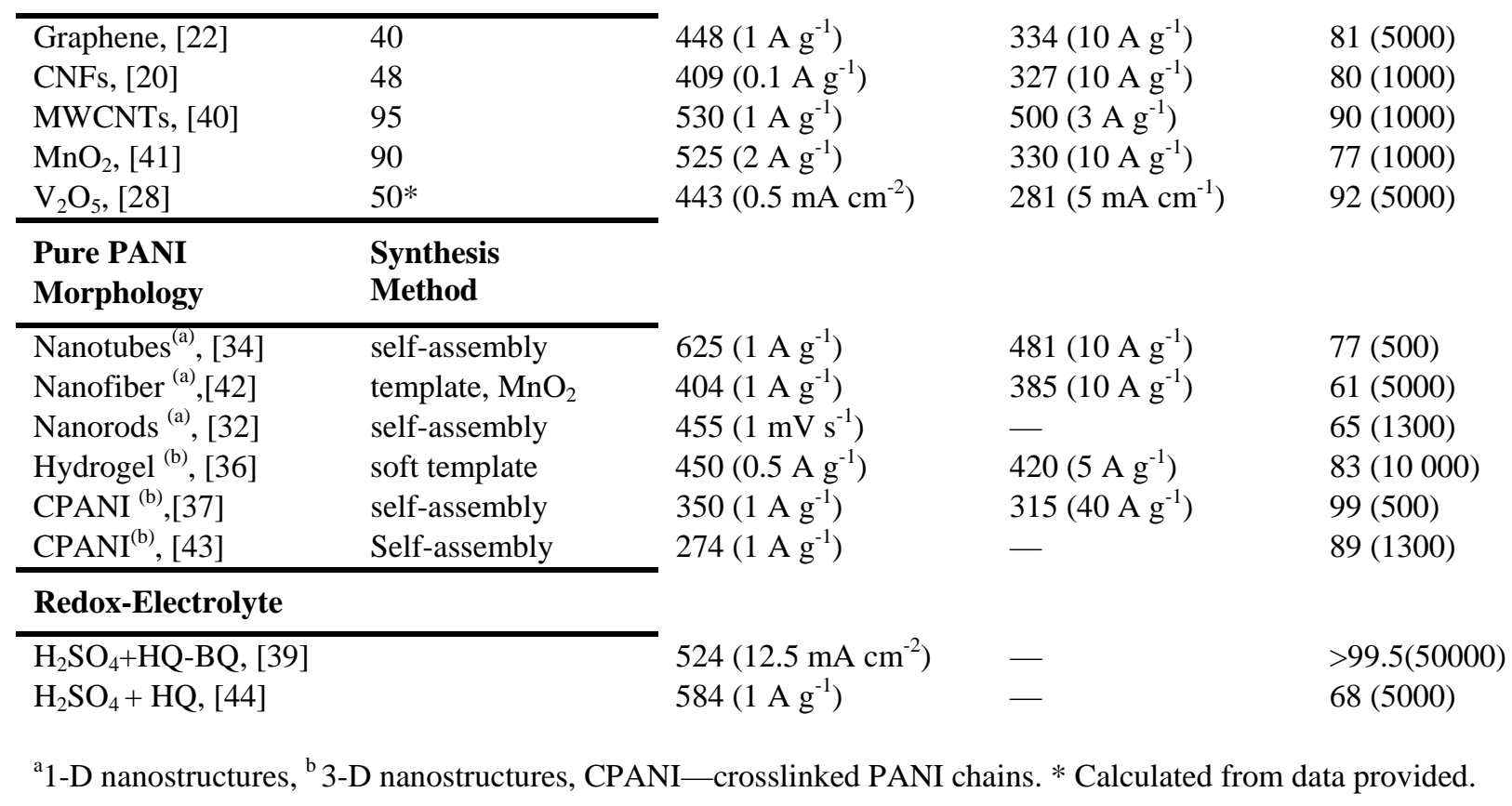

\section{Lithium-Ion batteries}

Demand for high energy and power density energy devices for large scale application such as grid-level energy storage and transportation calls for development of next generation lithium ion batteries and/or beyond lithium ion batteries. The state of the art lithium-ion batteries face challenges such as poor safety, high production cost and limited energy density of the electrode materials (the practical capacity of graphite is $\sim 300 \mathrm{mAhg}^{-1}$ while that of the $\mathrm{LiCoO}_{2}$ is $\sim 140$ $\mathrm{mAhg}^{-1}$ ).[45] Nanostructured inorganic electrode materials such as copper vanadates/oxides, silicon, $\mathrm{MoS}_{2}$, and $\mathrm{LiVPO}_{4}$ with high energy storage capability are being explored for nextgeneration Li-ion batteries.[46-48] However, their performance is hampered by poor electrical conductivities and short lifespan.[49] The poor cyclability is attributed to large volumetric changes due to intercalation/de-intercalation of Li ions, which lead to distortion of the inorganic nanomaterial morphology. Hierarchical PANI/inorganic nanocomposite has proven to be one plausible solution to address these deficiencies.[50-52] PANI (used in both the anode and cathode of Li-ion) enhances the electrical conductivity and provides buffer to accommodate volumetric expansion of the aforementioned inorganic materials. Sengodu et al recently reviewed the application of conducting polymers in Li-ion batteries and the reader is directed to this review article for the role of PANI in next generation Li-ion batteries. [53]

\section{Beyond lithium ion batteries: Role of PANI}

\section{Sodium ions batteries (SIBs)}

Sodium is an abundant and low cost element with similar physical and chemical characteristics as Li.[54] Although SIBs possess lower gravimetric capacity compared to LIBs (1169 $\mathrm{mAhg}^{-1}$ for Na vs. $3829 \mathrm{mAhg}^{-1}$ for Li), SIBs are considered suitable alternatives to LIBs on the basis of cost, safety and sustainability. SIBs rely on cation intercalation/de-intercalation mechanisms 
similar to those of LIBs. The progress of SIBs, however, has been thwarted by lack of appropriate active materials for both anode and cathode. $\mathrm{Na}^{+}$is $\sim 40 \%$ larger in diameter than $\mathrm{Li}^{+}$, which makes it difficult to find a host material with sufficiently large interstitial spacing to host $\mathrm{Na}^{+}$and allow reversible insertion/extraction process.[55] Hard carbon, inorganic compounds such as transition metal oxides and redox organic materials have been studied for SIB electrodes.[56-58] These electrode materials have been extensively reviewed elsewhere.[59,60] The extent of discussion in this section will look into recent studies involving application of PANI in SIB electrodes either as auxiliary material to promote electroactivity of the aforementioned electrode materials or as a potential independent electrode host material for $\mathrm{Na}$ ions.

\section{SIB Anode}

PANI has been utilized in SIB anodes as a supplementary material to promote electrical conductivity and structural stability of inorganic compounds such as tin dioxide and cobalt sulfide. These inorganic compounds are promising anode materials for Na-ion batteries due to their low cost, minimal environmental pollution and high theoretical capacity.[61] However, their application is jeopardized by poor cycle life caused by large volume expansion during insertion and extraction of the large $\mathrm{Na}^{+}$and low electrical conductivity. The repeated volume changes during cycling cause degradation of nanostructures and agglomeration of nanoparticles thus impeding electrolyte penetration and ion transport. Core-shell nanostructures of $\mathrm{SnO}_{2}$ hollow sphere-polyaniline composite ( $\mathrm{SnO}_{2}$-HS@PANI) [62] delivered reversible capacity of $213.5 \mathrm{mAhg}^{-1}$ after 400 cycles at $300 \mathrm{~mA} \mathrm{~g}^{-1}$ while the PANI-free $\mathrm{SnO}_{2}$ hollow spheres $\left(\mathrm{SnO}_{2}\right.$ HS) and bare $\mathrm{SnO}_{2}$ particle anodes retained only 145 and $14.4 \mathrm{mAhg}^{-1}$, respectively, after 400 cycles at the same current density. The $\mathrm{SnO}_{2} @ P A N I$ nanocomposite also exhibited higher capacity retention compared to $\mathrm{SnO}_{2} @$ carbon nanotubes and $\mathrm{SnO}_{2} /$ reduced graphene. [61,63] Similar improvement in performance was observed for $\mathrm{Co}_{3} \mathrm{~S}_{4} @ P A N I$ nanotube composites (see figure 3), [64] which exhibited a capacity retention of $252.5 \mathrm{mAhg}^{-1}$ and $170.1 \mathrm{mAhg}^{-1}$ at high

current rate of $4000 \mathrm{~mA} \mathrm{~g}^{-1}$ after 100 and 400 cycles, respectively. In comparison, the bare $\mathrm{Co}_{3} \mathrm{~S}_{4}$ anode only retained $58.2 \mathrm{mAhg}^{-1}$ after 100 cycles.

\section{SIB Cathode}

PANI is favorable as an active SIB cathode material over inorganic compounds on the basis of availability and flexibility of its framework (for rapid sodiation and de-sodiation). Its application, however, is limited by low doping/de-doping level and lack of Na storage capability, especially for the case of Na deficient anode. Na-rich PANI cathode, poly(aniline-co-aminobenzenesulfonic sodium) (PANS), was recently developed by grafting electron withdrawing $-\mathrm{SO}_{3} \mathrm{Na}$ groups on PANI chains and investigated for SIB application.[65] By grafting sulfonic groups on PANI chains, $\mathrm{Na}^{+}$can be inserted and extracted from the polymer chain during charge and discharge, respectively, therefore changing the mechanism of operation from the convectional doping/dedoping to insertion/extraction. The self-doping, PANS cathode delivered a reversible capacity of $133 \mathrm{mAh} \mathrm{g}^{-1}$ with an excellent capacity retention of $\sim 97 \%$ after 200 cycles. The PANS cathode also showed an impressive rate capability possibly due to the flexible polymer framework, which offers less resistance to insertion/extraction of $\mathrm{Na}^{+}$. Electron withdrawing o-nitroaniline has also 
been grafted onto PANI chains and tested as a cathode for SIBs.[66] The aniline-nitroaniline copolymer delivered a capacity of $180 \mathrm{mAhg}^{-1}$ at an average potential of $3.2 \mathrm{vs} \mathrm{Na} / \mathrm{Na}^{+}$and a reversible capacity of $173 \mathrm{mAhg}^{-1}$ after 50 cycles.

\section{Lithium sulfur batteries}

Lithium-Sulfur (LiS) batteries are attractive energy storage devices for large scale applications due to the natural abundance of sulfur and its high theoretical energy density (2600 vs. 400 $\mathrm{Whkg}^{-1}$ for Li-ion).[67] In LiS batteries, lithium is oxidized at the anode while sulfur is reduced at the cathode through a series of reactions with $\mathrm{Li}^{+}$to form $\mathrm{Li}_{2} \mathrm{~S}$. Commercialization of LiS, however, has been hampered by low sulfur utilization and poor cycle life. [68] While the low sulfur utilization is a results of its insulating nature, the low battery cyclability is caused by the dissolution and shuttling of the intermediate lithium polysulfides $\left(\mathrm{Li}_{2} \mathrm{~S}_{\mathrm{n}}, 2<\mathrm{n}<8\right)$ and volumetric changes during cycling [69]. Sulfur composites with various carbon materials and conducting polymers have been developed to address these challenges by promoting electrical conductivity and encapsulating sulfur and hence the polysulfides. Carbon/sulfur composites have been well studied and their advantages/limitations delineated.[68,70] PANI has been favorably studied for application in LiS cathodes due its processability at room temperature, strong affinity for the intermediate polysulfide and flexible framework which allows for sulfur volumetric expansion observed during life cycle performance tests.

The positive charges induced on PANI amino groups during acid doping promote electrostatic interaction between the polymer and the negatively-charged polysulfides $\left(\mathrm{S}_{\mathrm{n}}{ }^{2-}\right)$ during the charge-discharge process. Such interactions possibly result in chemical immobilization of the polysulfides, thereby limiting dissolution and migration of these intermediate species away from the cathode. Gao and co-workers prepared ultrathin sulfur-wrapped PANI nanofibers with coreshell morphology via heterogonous nucleation of sulfur on water dispersed PANI nanofibers.[71] The PANI@sulfur nanofiber cathodes showed an initial discharge capacity of $977 \mathrm{mAh} \mathrm{g}^{-1}$ with over $83 \%$ retention after 100 cycles at $1 \mathrm{C}$ and excellent rate capability up to $3 \mathrm{C}$.

To more effectively impede dissolution of polysulfides and to also accommodate volume changes, several works have incorporated a heating (vulcanization) step during the fabrication of PANI/sulfur nanocomposites with tubular, hollow-spherical and yolk-shell morphologies.[72-74] The heat treatment process results in a small amount of $S$ reacting with the polymer backbone giving rise to a 3-D, crosslinked sulfur-polymer backbone with the inter- and intra-chain disulfide bonds interconnected via vulcanization.[73] The unreacted S and polysulfides are physically and chemically trapped within the polymer molecular framework. Furthermore, the void spaces created due to the polymer-sulfur reaction provide breathing room to accommodate volume changes associated with Li/S electrochemistry. Hollow sulfur@PANI spheres recently demonstrated a reversible discharge capacity of $602 \mathrm{mAhg}^{-1}$ after 1000 cycles at 0.5C.[72]

Several recent works have developed and investigated layered ternary composites of PANI/sulfur/carbon as LiS cathodes with the aim to combine the high mechanical strength and electrical conductivity of carbon with the enhanced sulfur confinement by PANI. Two 
fabrication methods have been adapted for such ternary composites based on the sequence of sulfur or PANI deposition on the carbon substrate. The first approach involves the deposition of sulfur on a high surface area carbon nanomaterial and coating of the resultant bilayer system with a thin layer of PANI to encapsulate sulfur and prevent out-diffusion of the polysulfides. This approach was shown by Kim et al who developed a freestanding PANI/sulfur/single wall carbon nanotube (PANI/S/SWNT) film as illustrated in figure 4.[75] The composite cathode showed initial discharge specific capacity of $1415 \mathrm{mAhg}^{-1}$ with retention of $1011 \mathrm{mAhg}^{-1}$ after 100 cycles at $0.2 \mathrm{C}$. The second approach entails polymerizing a thin layer of PANI on the surface/pores of a carbon material followed by deposition of sulfur. The PANI layer is intended to foster chemical interaction between sulfur and the underlying high surface area carbon. LiS cathodes based on sulfur/PANI/mesoporous carbon reported the highest discharge capacity of $675 \mathrm{mAhg}^{-1}$ after 200 cycles at 0.1 C.[76] PANI provided a soft padding between carbon and sulfur, reducing detachment of sulfur and lithium polysulfide within the meso-pores of the carbon material during cycling.

Ultrathin composite film of PANI/MWCTs has also been recently developed to serve as an interlayer between the separator and the LiS cathode. Chang et al [77] functionalized one side of the separator facing the cathode with ultralow loading of $0.01 \mathrm{mg} \mathrm{cm}^{-2}$ of PANI nanofibers and multiwalled carbon nanotubes (PANiNFs/MWCNT) and investigated its performance in LiS batteries. The interlayer facilitated reversible capacities of 709, 641 and $612 \mathrm{mAhg}^{-1}$, respectively, at C/5, C/2 and $1 \mathrm{C}$ after 100 cycles. The higher utilization of sulfur was attributed to the additional electron pathway provided by the PANI-based coating ensuring accessibility of the active material. The enhanced cyclability was due to the PANiNF/MWCNT filter blocking the polysulfides via chemical interaction with the PANI imine groups as well as via reactivation of the trapped species by the conductive separator.

\section{Summary and perspective}

This review provides a brief overview of the present status of the application of PANI and its composites in the electrodes of supercapacitors, sodium-ion and lithium-sulfur batteries. Although satisfactory research progress has been realized both in supercapacitors and the covered rechargeable batteries, much fundamental studies and electrode fabrication techniques remains to be done. One of the areas that can be further improved in the fabrication of electrodes is the development of binder-free and freestanding electrodes. Few freestanding film electrodes based on PANI hybrids have been reported with good electrochemical performance.[16,20] However, most electrodes in the literature are prepared by making a paste consisting of the active materials, binders and conductive materials, which is then coated on a current collector. Such rigorous process is likely to cause impairment to the fabricated PANI-based nanostructures. The continuity of electron transport pathways and/or extent of encapsulation/coating of active materials for the battery configurations will most likely be interrupted. Furthermore, by incorporation of binders, additional dead weight is introduced to the system. The long-term stability of PANI-based electrodes needs to be enhanced for commercialization of these energy devices (particularly supercapacitors). In light of recent impressive electroactivity and stability of PANI-based electrodes demonstrated in redox-active electrolytes where capacitance retention 
was observed over 50k cycles [39], further research on nanostructured PANI/PANI hybrid electrode in such and other electrolyte systems [78] might prove invaluable in enhancing electrode stability.

There is a need for fundamental in situ studies on aspects such as PANI redox state, conductivity, structural integrity and chemical interaction of PANI with active material and/or unstable intermediate species during battery cycling, where PANI is largely added for either electrical conductivity and/or interaction with active species. The redox state of PANI, and consequently its conductivity, is highly dependent on electrode potential. Emeraldine is the most conductive state and hence the most appropriate state for electrode application where high electron conductivity is paramount. It will be therefore insightful to study the extent of PANI redox process, if any, and its reversibility during charge-discharge process since such process might impact negatively on electrode electroactivity/stability. Moreover, it will be invaluable to decipher the extent of chemical interaction between PANI and sulfur/intermediate polysulfide and effect of ions consumptions to such interaction during charge-discharge process for the case of LiS batteries.

There has also been some oversight in application of PANI in LiS electrodes - electrode fabrication processes such as heat treatment (vulcanization process) is likely to cause degradation of dopants and therefore diminish PANI conductivity significantly. Most literature works have also not studied the capacity arising from the cation doping/de-doping of PANI chains in battery electrodes that may have resulted in overestimation of the capacity of the active material(s).

\section{Acknowledgements}

Authors would like to thank National Science Foundation (award numbers: CMMI 1537827 and CBET 1236466) for financial support.

\section{References}

Papers of particular interest, published within the period of review, have been highlighted as:

\section{* of special interest}

\section{** of outstanding interest}

1. Bruce PG, Freunberger SA, Hardwick LJ, Tarascon JM: Li-O2 and Li-S batteries with high energy storage. Nat Mater 2012, 11:19-29.

* Good review on new generation of Li based batteries (Li-S and Li-Air)

2. Choi NS, Chen Z, Freunberger SA, Ji X, Sun YK, Amine K, Yushin G, Nazar LF, Cho J, Bruce PG: Challenges facing lithium batteries and electrical double-layer capacitors. Angew Chem Int Ed Engl 2012, 51:9994-10024. 
3. Slater MD, Kim D, Lee E, Johnson CS: Sodium-Ion Batteries. Adv Funct Mater 2013, 23:947-958.

4. MacDiarmid AG: Polyanilines: A Novel Class of Conducting Polymers. Farad Disc Chem Soc 1989, 88:317-332.

5. Chiang J, Macdiarmid AG: Polyaniline: Protonic acid doping of the emearaldine form to the metalic regime. Synthetic Metals 1986, 13:193-205.

6. Xia Y, MacDiarmid AG: Camphorsulfonic Acid Fully Doped Polyaniline Emeraldine Salt: In Situ Observation of Electronic and Conformational Changes Induced by Organic Vapors by an Ultraviolet/ Visible/Near-Infrared Spectroscopic Method. Macromolecules 1994, 27:7212-7214.

7. Long Y-Z, Li M-M, Gu C, Wan M, Duvail J-L, Liu Z, Fan Z: Recent advances in synthesis, physical properties and applications of conducting polymer nanotubes and nanofibers. Progress in Polymer Science 2011, 36:1415-1442.

8. Tran HD, D'Arcy JM, Wang Y, Beltramo PJ, Strong VA, Kaner RB: The oxidation of aniline to produce "polyaniline": a process yielding many different nanoscale structures. $J$. Mater. Chem. 2011, 21:3534-3550.

9. Li H, Wang J, Chu Q, Wang Z, Zhang F, Wang S: Theoretical and experimental specific capacitance of polyaniline in sulfuric acid. $J$ Power Sources 2009, 190:578-586.

10. Miao YE, Fan W, Chen D, Liu T: High-performance supercapacitors based on hollow polyaniline nanofibers by electrospinning. ACS. Appl. Mater. Interfaces. 2013, 5:44234428.

* Authors report fabrication of hollow PANI nanofibers with controllable wall thickness via insitu deposition of PANI on sacrificial electrospun template. The hollow nanofibers exhibit excellent electrochemical performance at $1 \mathrm{~A} \mathrm{~g}^{-1}$.

11. Luo Y, Kong D, Jia Y, Luo J, Lu Y, Zhang D, Qiu K, Li CM, Yu T: Self-assembled graphene@PANI nanoworm composites with enhanced supercapacitor performance. RSC Advances 2013, 3:5851-5859.

12. Wu W, Pan D, Li Y, Zhao G, Jing L, Chen S: Facile fabrication of polyaniline nanotubes using the self-assembly behavior based on the hydrogen bonding: a mechanistic study and application in high-performance electrochemical supercapacitor electrode. Electrochim Acta 2015, 152:126-134.

*This work demonstrates fabrication of 1-D PANI nanotubes with excellent electroactivity via template-less process.

13. Wang K, Wu H, Meng Y, Wei Z: Conducting polymer nanowire arrays for high performance supercapacitors. Small 2014, 10:14-31.

14. Beguin F, Presser V, Balducci A, Frackowiak E: Carbons and electrolytes for advanced supercapacitors. Adv Mater 2014, 26:2219-2251.

15. Zhou Z, Wu X-F, Hou H: Electrospun carbon nanofibers surface-grown with carbon nanotubes and polyaniline for use as high-performance electrode materials of supercapacitors. RSC Advances 2014, 4:23622-23629.

16. Meng Y, Wang K, Zhang Y, Wei Z: Hierarchical porous graphene/polyaniline composite film with superior rate performance for flexible supercapacitors. Adv Mater 2013, 25:6985-6990. 
17. Jingjing Xu, Kai Wang, Sheng-Zhen Zu, Bao-Hang Han, Zhixiang Wei: Hierarchical Nanocomposites of Polyaniline Nanowire Arrays on Graphene Oxide Sheets with Synergistic Effect for Energy Storage. 2010, 4:5019-5026.

18. Wang L, Lu X, Lei S, Song Y: Graphene-based polyaniline nanocomposites: preparation, properties and applications. J Mater Chem A 2014, 2:4491-4509.

** Review provides excellent discussion on the synthesis of PANI/Graphene nanomposites and application in supercapacitors among others.

19. Ashok Kumar N, Baek JB: Electrochemical supercapacitors from conducting polyaniline-graphene platforms. Chem Commun (Camb) 2014, 50:6298-6308.

20. Tran C, Singhal R, Lawrence D, Kalra V: Polyaniline-coated freestanding porous carbon nanofibers as efficient hybrid electrodes for supercapacitors. J Power Sources 2015, 293:373-379.

*Paper demonstrates fabrication of freestanding PANI-coated hierarchical carbon nanofibers for supercapacitor application.

21. Li L, Raji AR, Fei H, Yang Y, Samuel EL, Tour JM: Nanocomposite of polyaniline nanorods grown on graphene nanoribbons for highly capacitive pseudocapacitors. ACS Appl Mater Interfaces 2013, 5:6622-6627.

22. Hassan M, Reddy KR, Haque E, Faisal SN, Ghasemi S, I. Minett A, Gomes VG: Hierarchical assembly of graphene/polyaniline nanostructures to synthesize freestanding supercapacitor electrode. Composites Sci Technol 2014, 98:1-8.

23. Wei J, Zhang J, Liu Y, Xu G, Chen Z, Xu Q: Controlled growth of whisker-like polyaniline on carbon nanofibers and their long cycle life for supercapacitors. RSC Advances 2013, 3:3957-3962.

24. Zheng X, Luo J, Lv W, Wang DW, Yang QH: Two-Dimensional Porous Carbon: Synthesis and Ion-Transport Properties. Adv Mater 2015, 27:5388-5395.

25. Lin H, Li L, Ren J, Cai Z, Qiu L, Yang Z, Peng H: Conducting polymer composite film incorporated with aligned carbon nanotubes for transparent, flexible and efficient supercapacitor. Sci Rep 2013, 3:1-5.

26. Xie K, Li J, Lai Y, Zhang Z, Liu Y, Zhang G, Huang H: Polyaniline nanowire array encapsulated in titania nanotubes as a superior electrode for supercapacitors. Nanoscale 2011, 3:2202-2207.

27. Chen L, Song Z, Liu G, Qiu J, Yu C, Qin J, Ma L, Tian F, Liu W: Synthesis and electrochemical performance of polyaniline-MnO2 nanowire composites for supercapacitors. J Phys Chem Solids 2013, 74:360-365.

28. Bai M-H, Liu T-Y, Luan F, Li Y, Liu X-X: Electrodeposition of vanadium oxidepolyaniline composite nanowire electrodes for high energy density supercapacitors. J Mater Chem A 2014, 2:10882-10888.

*Demonstrates preparation of PANI-transition metal oxide nanocomposites with impressive electrochemical performance in supercapacitors.

29. Zhang P, Liu Z, Liu Y, Fan H, Jiao Y, Chen B: Titanium Dioxide@Polyaniline Core-Shell Nanowires as High-Performance and Stable Electrodes for Flexible Solid-State Supercapacitors. Electrochimica Acta 2015, 184:1-7. 
30. Kumar V, Lee PS: Redox Active Polyaniline-h-MoO3Hollow Nanorods for Improved Pseudocapacitive Performance. The J Physl Chem C 2015, 119:9041-9049.

31. Xu H, Li X, Wang G: Polyaniline nanofibers with a high specific surface area and an improved pore structure for supercapacitors. J Power Sources 2015, 294:16-21.

32. Wang X, Deng J, Duan X, Liu D, Guo J, Liu P: Crosslinked polyaniline nanorods with improved electrochemical performance as electrode material for supercapacitors. $J$ Mater Chem A 2014, 2:12323.

33. Shi Y, Peng L, Ding Y, Zhao Y, Yu G: Nanostructured conductive polymers for advanced energy storage. Chem Soc Rev 2015, 44:6684-6696.

**An excellent review on the synthesis and application of nanostructured conducting polymers in energy devices.

34. Mu J, Ma G, Peng H, Li J, Sun K, Lei Z: Facile fabrication of self-assembled polyaniline nanotubes doped with d-tartaric acid for high-performance supercapacitors. $J$ Power Sources 2013, 242:797-802.

35. Shi Y, Yu G: Designing Hierarchically Nanostructured Conductive Polymer Gels for Electrochemical Energy Storage and Conversion. Chemistry of Materials 2016, 28:2466-2477.

36. Pan L, Yu G, Zhai D, Lee HR, Zhao W, Liu N, Wang H, Tee BCK, Shi Y, Cui Y, et al.: Hierarchical nanostructured conducting polymer hydrogel with high electrochemical activity. PNAs 2012, 109:9287-9292.

37. Gawli Y, Banerjee A, Dhakras D, Deo M, Bulani D, Wadgaonkar P, Shelke M, Ogale S: 3D Polyaniline Architecture by Concurrent Inorganic and Organic Acid Doping for Superior and Robust High Rate Supercapacitor Performance. Sci Reports 2016, 6:21002-21011.

** Fabrication of highly conductive, crosslinked PANI nanostructures for supercapacitor application. Supercapacitor electrodes based on 3-D PANI architecture delivered unprecedent rate capability and good electrochemical stability.

38. Chen W, Xia C, Rakhi RB, Alshareef HN: A general approach toward enhancement of pseudocapacitive performance of conducting polymers by redox-active electrolytes. J Power Sources 2014, 267:521-526.

39. Vonlanthen D, Lazarev P, See KA, Wudl F, Heeger AJ: A stable polyanilinebenzoquinone-hydroquinone supercapacitor. Adv Mater 2014, 26:5095-5100.

**This work demonstrates outstanding electrochemical stability of PANI based supercapacitors with incoporation of redox-active species, hyrdoquinone-benzoquinone, to conventional electrolyte $\left(\mathrm{H}_{2} \mathrm{SO}_{4} /\right.$ Acetic acid).

40. Chang C, Weng C, Chien C, Chuang T, TingYin Lee JY, Weib Y: Polyaniline/carbon nanotube nanocomposite electrodes with biomimetic hierarchical structure for supercapacitors. J. Mater. Chem. A. 2013, 1:14719-14728.

41. Ansari SA, Parveen N, Han TH, Ansari MO, Cho MH: Fibrous polyaniline@manganese oxide nanocomposites as supercapacitor electrode materials and cathode catalysts for improved power production in microbial fuel cells. Phys Chem Chem Phys 2016, 18:9053-9060. 
42. Chen W, Rakhi RB, Alshareef HN: Morphology-Dependent Enhancement of the Pseudocapacitance of Template-Guided Tunable Polyaniline Nanostructures. The $J$ Phys Chem C 2013, 117:15009-15019.

43. Wang X, Liu D, Deng J, Duan X, Guo J, Liu P: Improving cyclic stability of polyaniline by thermal crosslinking as electrode material for supercapacitors. RSC Adv. 2015, 5:78545-78552.

44. Xie H, Zhu Y, Wu Y, Wu Z, Liu E: The effect of hydroquinone as an electrolyte additive on electrochemical performance of the polyaniline supercapacitor. Mater Res Bulletin 2014, 50:303-306.

45. Reddy MV, Subba Rao GV, Chowdari BV: Metal oxides and oxysalts as anode materials for Li ion batteries. Chem Rev 2013, 113:5364-5457.

46. Goriparti S, Miele E, De Angelis F, Di Fabrizio E, Proietti Zaccaria R, Capiglia C: Review on recent progress of nanostructured anode materials for $\mathrm{Li}$-ion batteries. Journal of Power Sources 2014, 257:421-443.

47. Lepage D, Michot C, Liang G, Gauthier M, Schougaard SB: A soft chemistry approach to coating of LiFePO4 with a conducting polymer. Angew Chem Int Ed Engl 2011, 50:6884-6887.

48. Zhang Q, Wang J, Xu D, Wang Z, Li X, Zhang K: Facile large-scale synthesis of vertically aligned CuO nanowires on nickel foam: growth mechanism and remarkable electrochemical performance. Journal of Materials Chemistry A 2014, 2:3865-3874.

49. Hu L, Ren Y, Yang H, Xu Q: Fabrication of 3D hierarchical MoS(2)/polyaniline and $\operatorname{MoS}(2) / C$ architectures for lithium-ion battery applications. ACS Appl Mater Interfaces 2014, 6:14644-14652.

50. Xue Q, Li J, Xu G, Zhou H, Wang X, Kang F: In situ polyaniline modified cathode material Li[Li0.2Mn0.54Ni0.13Co0.13]O2with high rate capacity for lithium ion batteries. J. Mater. Chem. A 2014, 2:18613-18623.

51. Wu H, Yu G, Pan L, Liu N, McDowell MT, Bao Z, Cui Y: Stable Li-ion battery anodes by in-situ polymerization of conducting hydrogel to conformally coat silicon nanoparticles. Nat Commun 2013, 4:1943-1948.

52. Kummer M, Badillo JP, Schmitz A, Bremes HG, Winter M, Schulz C, Wiggers H: Silicon/Polyaniline Nanocomposites as Anode Material for Lithium Ion Batteries. Journal of the Electrochemical Society 2013, 161:A40-A45.

53. Sengodu P, Deshmukh AD: Conducting polymers and their inorganic composites for advanced Li-ion batteries: a review. RSC Adv. 2015, 5:42109-42130.

54. Pan H, Hu Y-S, Chen L: Room-temperature stationary sodium-ion batteries for largescale electric energy storage. Energy \& Environl Sci 2013, 6:2338-2360.

55. Cao Y, Xiao L, Sushko ML, Wang W, Schwenzer B, Xiao J, Nie Z, Saraf LV, Yang Z, Liu J: Sodium ion insertion in hollow carbon nanowires for battery applications. Nano Lett 2012, 12:3783-3787.

56. Xia X, Dahn JR: Study of the Reactivity of NaHard Carbon with Different Solvents and Electrolytes. J Electrochem Soc 2012, 159:A515-A519.

57. Xiong H, Slater MD, Balasubramanian M, Johnson CS, Rajh T: Amorphous TiO2Nanotube Anode for Rechargeable Sodium Ion Batteries. The J Phys Chem Lett 2011, 2:25602565. 
58. Xiao L, Cao Y, Xiao J, Wang W, Kovarik L, Nie Z, Liu J: High capacity, reversible alloying reactions in $\mathrm{SnSb} / \mathrm{C}$ nanocomposites for Na-ion battery applications. Chem Commun (Camb) 2012, 48:3321-3323.

59. Slater MD, Kim D, Lee E, Johnson CS: Sodium-Ion Batteries. Advanced Functional Materials 2013, 23:947-958.

**Review on SIBs, providing insightful comparison with LIBs and potential electrode/electrolyte materials for SIBs.

60. Tang J, Dysart AD, Pol VG: Advancement in sodium-ion rechargeable batteries. Current Opinion in Chem Engn 2015, 9:34-41.

61. Wang Y-X, Lim Y-G, Park M-S, Chou S-L, Kim JH, Liu H-K, Dou S-X, Kim Y-J: Ultrafine SnO2nanoparticle loading onto reduced graphene oxide as anodes for sodium-ion batteries with superior rate and cycling performances. J. Mater. Chem. A 2014, 2:529-534.

62. Zhao X, Zhang Z, Yang F, Fu Y, Lai Y, Li J: Core-shell structured SnO2hollow spherespolyaniline composite as an anode for sodium-ion batteries. RSC Adv. 2015, 5:3146531471.

63. Wang Y, Su D, Wang C, Wang G: SnO2@MWCNT nanocomposite as a high capacity anode material for sodium-ion batteries. Electrochem Comm 2013, 29:8-11.

64. Zhou Q, Liu L, Huang Z, Yi L, Wang X, Cao G: Co3S4@polyaniline nanotubes as highperformance anode materials for sodium ion batteries. J. Mater. Chem. A 2016, 4:5505-5516.

65. Zhou M, Li W, Gu T, Wang K, Cheng S, Jiang K: A sulfonated polyaniline with high density and high rate Na-storage performances as a flexible organic cathode for sodium ion batteries. Chem Commun (Camb) 2015, 51:14354-14356.

*Excellent demonstration of modified PANI as low cost material for Na ion storage in SIB cathodes.

66. Zhao R, Zhu L, Cao Y, Ai X, Yang HX: An aniline-nitroaniline copolymer as a high capacity cathode for Na-ion batteries. Electrochem Comm 2012, 21:36-38.

67. Zheng G, Yang Y, Cha JJ, Hong SS, Cui Y: Hollow carbon nanofiber-encapsulated sulfur cathodes for high specific capacity rechargeable lithium batteries. Nano Lett 2011, 11:4462-4467.

68. Cheng H, Wang S: Recent progress in polymer/sulphur composites as cathodes for rechargeable lithium-sulphur batteries. J Mater Chem A 2014, 2:13783-13794.

69. Wu F, Chen J, Li L, Zhao T, Chen R: Improvement of Rate and Cycle Performence by Rapid Polyaniline Coating of a MWCNT/Sulfur Cathode. The Phys Chem C 2011, 115:24411-24417.

70. Park JS, Cho GB, Ryu HS, Ahn JH, Ahn HJ, Kim KW: Sulphur-carbon composites for Li/S batteries. Materials Technology 2013, 28:270-275.

71. Gao H, Lu Q, Liu N, Wang X, Wang F: Facile preparation of an ultrathin sulfur-wrapped polyaniline nanofiber composite with a core-shell structure as a high performance cathode material for lithium-sulfur batteries. J. Mater. Chem. A 2015, 3:7215-7218.

72. Ma G, Wen Z, Jin J, Lu Y, Wu X, Wu M, Chen C: Hollow polyaniline sphere@sulfur composites for prolonged cycling stability of lithium-sulfur batteries. J Mater Chem A 2014, 2:10350-10354. 
**This work demonstrates effectiveness of PANI to encapsulate sulfur and contain outdiffussion of unstable intermediate polysulfides leading to excellent cycle life of LiS cathodes. Capacity retention of $602 \mathrm{mAhg}^{-1}$ was observed after 1000 cycles.

73. Xiao L, Cao Y, Xiao J, Schwenzer B, Engelhard MH, Saraf LV, Nie Z, Exarhos GJ, Liu J: A soft approach to encapsulate sulfur: polyaniline nanotubes for lithium-sulfur batteries with long cycle life. Adv Mater 2012, 24:1176-1181.

74. Xiao L, Cao Y, Xiao J, Schwenzer B, Engelhard MH, Saraf LV, Nie Z, Exarhos GJ, Liu J: Molecular structures of polymer/sulfur composites for lithium-sulfur batteries with long cycle life. J Mater Chem A 2013, 1:9517-9526.

75. Kim JH, Fu K, Choi J, Kil K, Kim J, Han X, Hu L, Paik U: Encapsulation of S/SWNT with PANI web for enhanced rate and cycle performance in lithium sulfur batteries. Sci Rep 2015, 5:8946-8951.

**This work reports fabrication of ternary composites of PANI-sulfur-SWNT with improved cycle life perfomance in LiS batteries.

76. Zhao X, Ahn H-J, Kim K-W, Cho K-K, Ahn J-H: Polyaniline-Coated Mesoporous Carbon/Sulfur Composites for Advanced Lithium Sulfur Batteries. The Phys Chem C 2015, 119:7996-8003.

77. Chang C-H, Chung S-H, Manthiram A: Ultra-lightweight PANiNF/MWCNTfunctionalized separators with synergistic suppression of polysulfide migration for Li-S batteries with pure sulfur cathodes. J. Mater. Chem. A 2015, 3:18829-18834.

**A composite of PANI and MWCNT was employed as an interalyer between LiS cathode and seperator to prevent migration of polysulfides to the anode.

78. Chen W, Rakhi RB, Alshareef HN: Facile synthesis of polyaniline nanotubes using reactive oxide templates for high energy density pseudocapacitors. J. Mater. Chem. A. 2013, 1:3315-3324. 


\section{Figures}

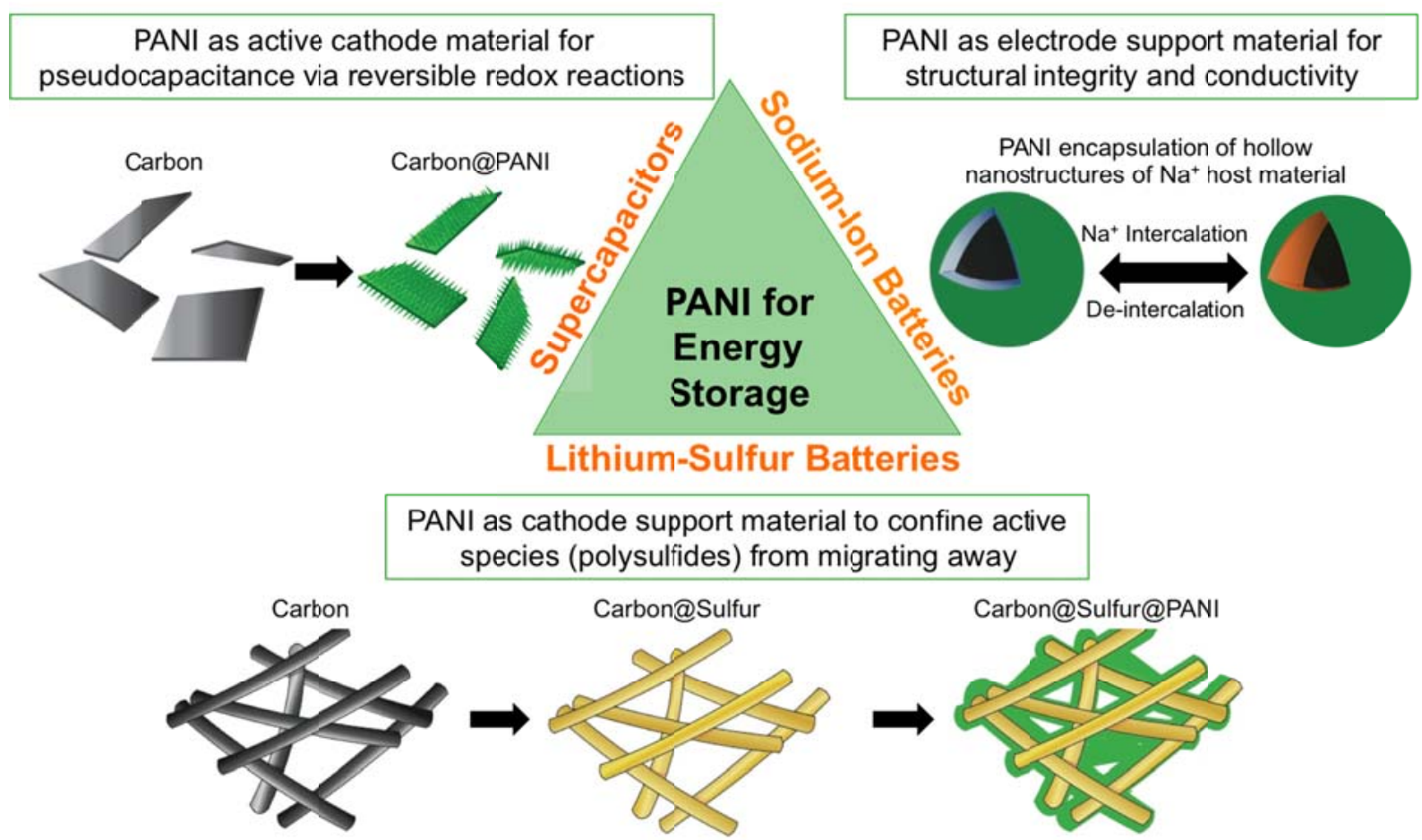

Figure 1: Schematic showing the versatility of polyaniline for applicaton in energy storage devices both as an active material and as a support material to enhance device capacity and/or stability. 

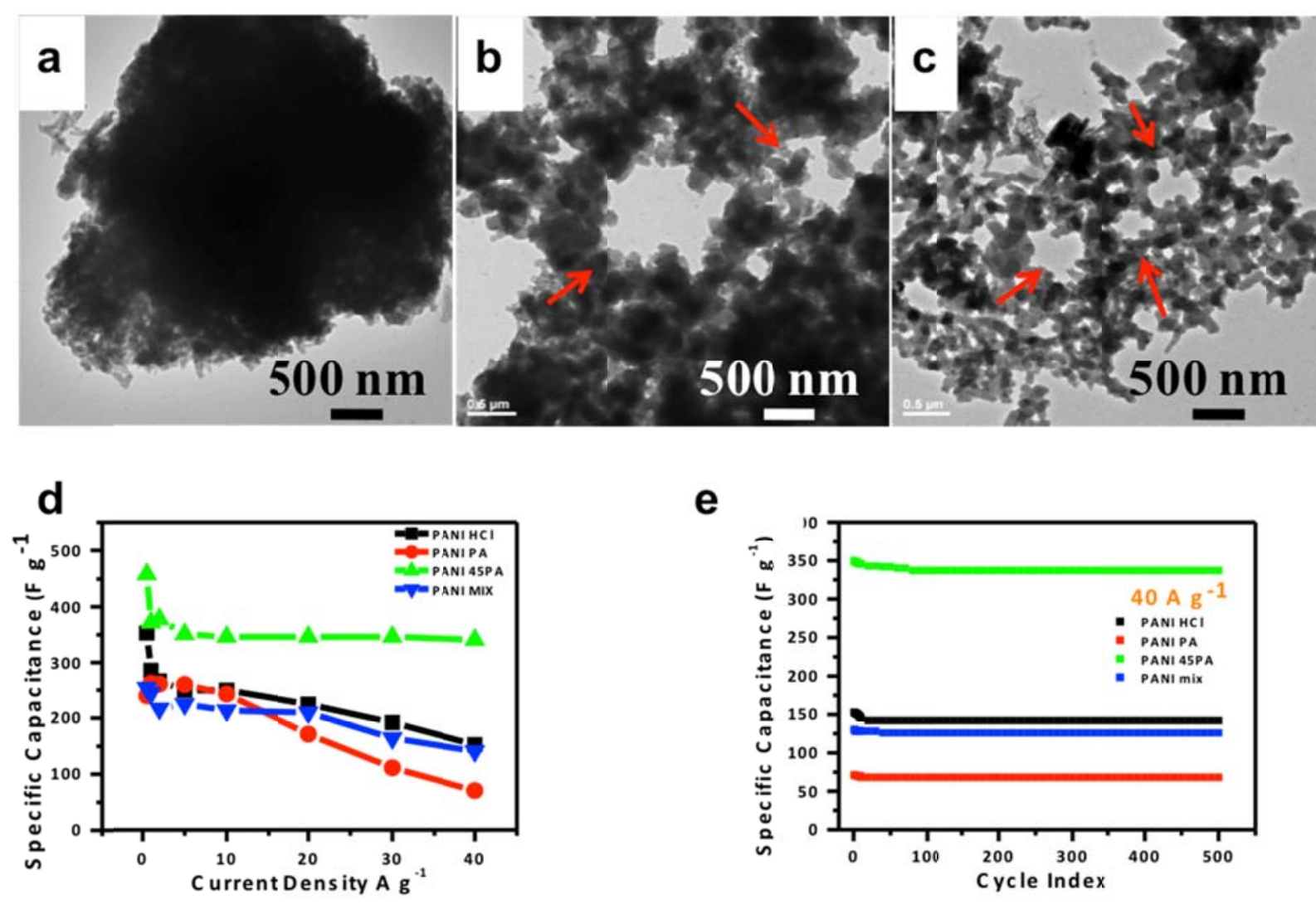

Figure 2: PANI application in supercapacitors: TEM images showing PANI doped by $\mathrm{HCl}$ (a), phytic acid (b) and crosslinked PANI obtained by concurrent-doping with HCl/phytic acid (c). Rate capability (d) and life cycle (e) for PANI electrodes obtained by doping PANI with different $\mathrm{HCl} /$ Phytic acid ratios. Best performance was obtained for co-doped PANI sample with 45\% phytic acid (PANI 45PA) which showed unprecedent rate capability upto $40 \mathrm{~A} / \mathrm{g}$. Reprinted with permission from Gawli Y, Banerjee A, Dhakras D, Deo M, Bulani D, Wadgaonkar P, Shelke M, Ogale S: 3D Polyaniline Architecture by Concurrent Inorganic and Organic Acid Doping for Superior and Robust High Rate Supercapacitor Performance. Copyright 2016 Nature Publishing Group. 

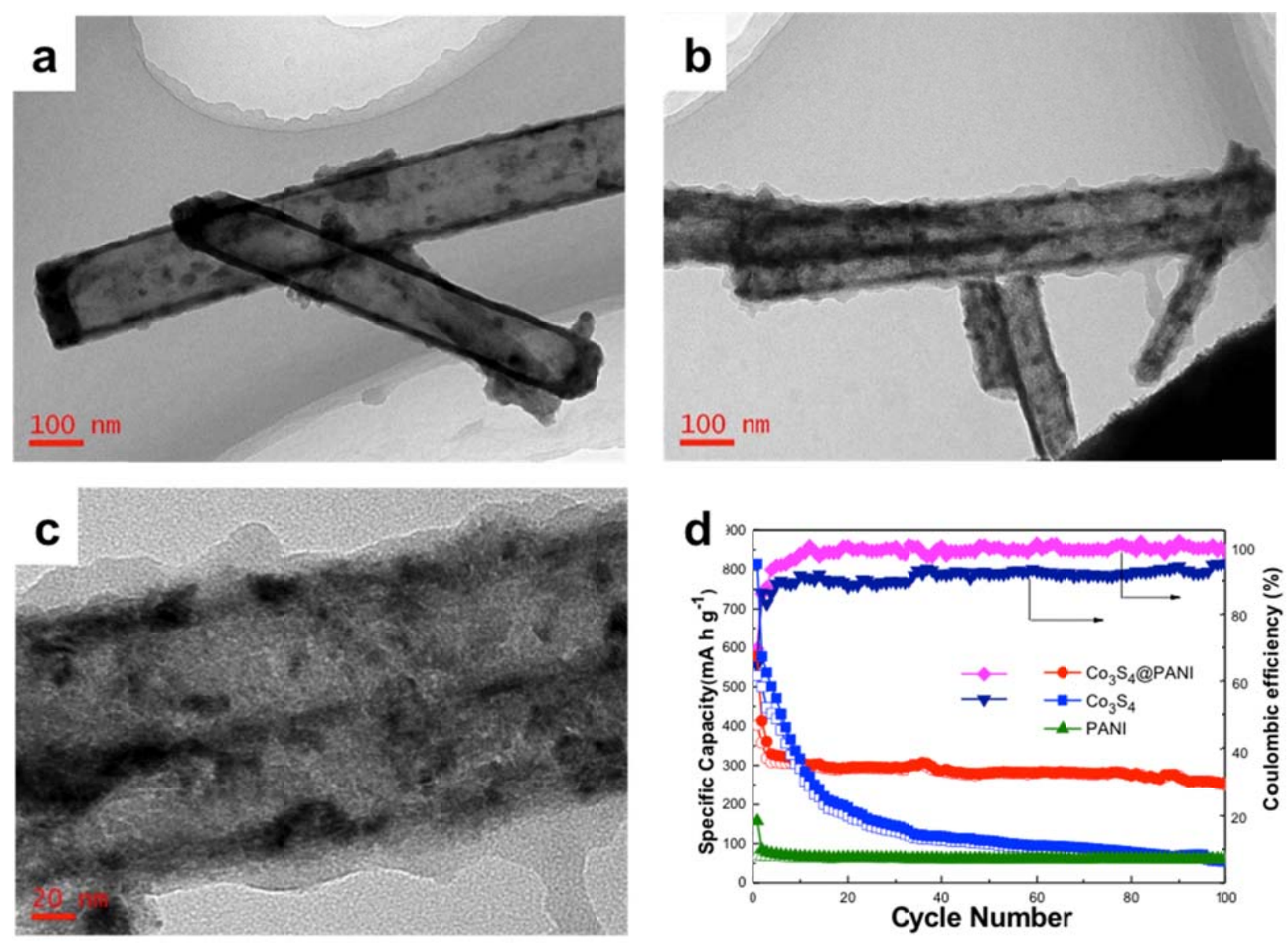

Figure 3: PANI application in sodium-ion batteries: TEM images of bare $\mathrm{Co}_{3} \mathrm{~S}_{4}$ nanotubes (a), PANI coated $\mathrm{Co}_{3} \mathrm{~S}_{4}$ nanotubes $\left(\mathrm{Co}_{3} \mathrm{~S}_{4} @ P A N I\right)(b)$, and $\mathrm{Co}_{3} \mathrm{~S}_{4} @ P A N I$ at higher magnification (c) and electrochemical performance (d) of PANI (green), $\mathrm{Co}_{3} \mathrm{~S}_{4}$ nanotubes (blue) and $\mathrm{Co}_{3} \mathrm{~S}_{4}$ @PANI nanotubes. Coating of $\mathrm{Co}_{3} \mathrm{~S}_{4}$ nanotubes with PANI is seen to enhance life cycle performance. Reprinted with permission from Zhou Q, Liu L, Huang Z, Yi L, Wang X, Cao G: $\mathrm{Co}_{3} \mathrm{~S}_{4} @$ polyaniline nanotubes as high-performance anode materials for sodium ion batteries. Images adapted with permission of The Royal Society of Chemistry. 


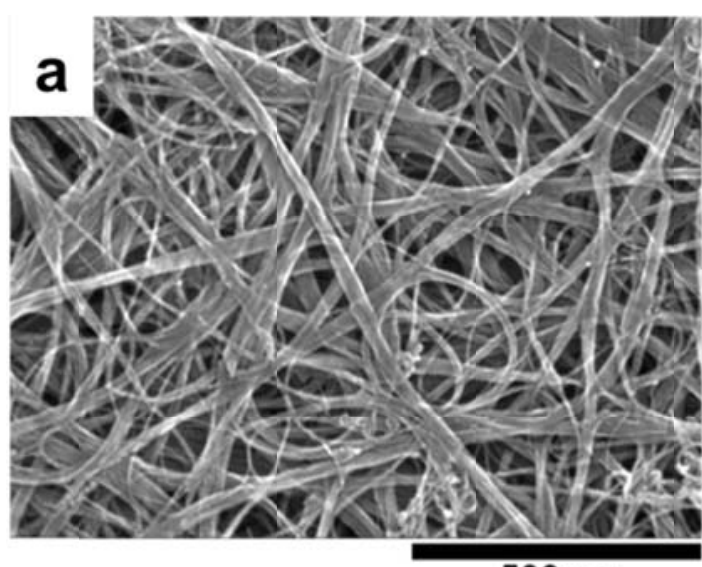

$500 \mathrm{~nm}$

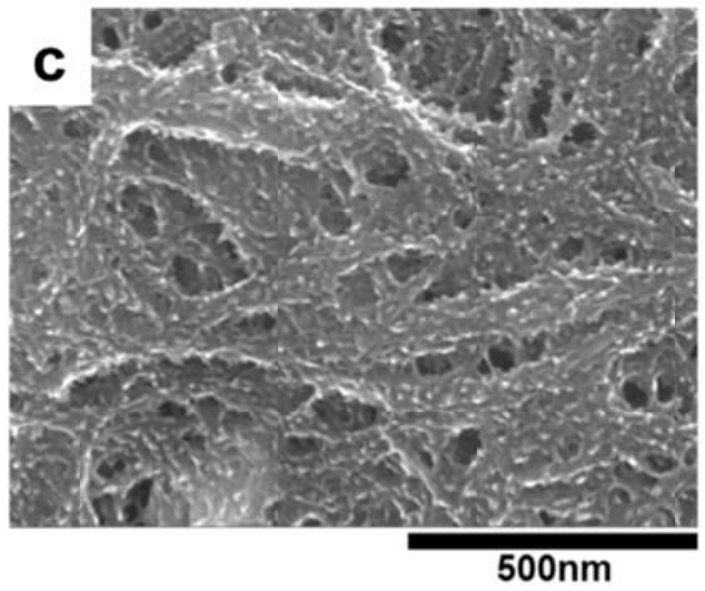

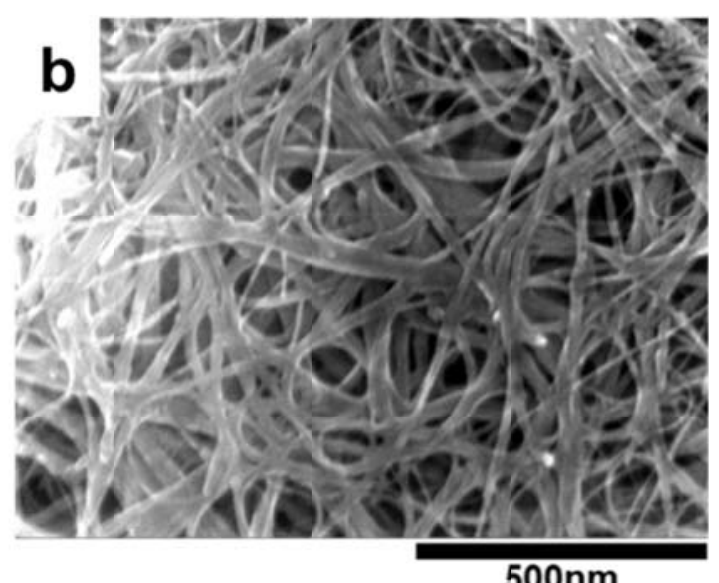

$500 \mathrm{~nm}$

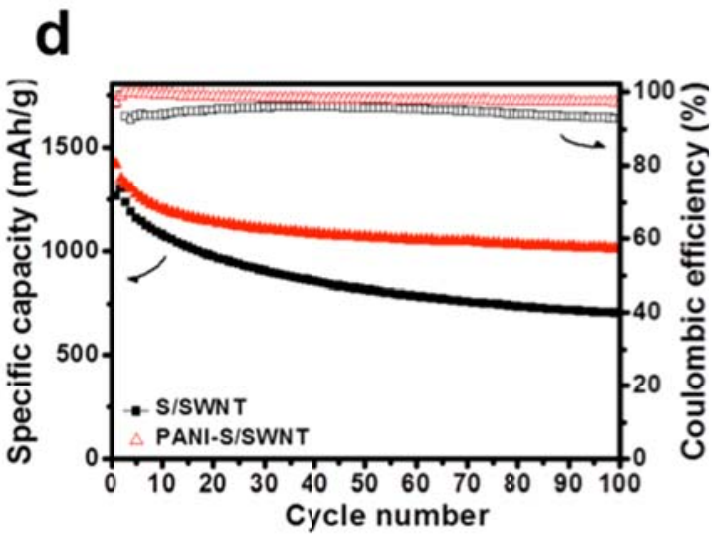

Figure 4: PANI application in Lithium-sulfur batteries: SEM images of freestanding SWNTs (a), sulfur deposited on SWNTs, S/SWNT (b), PANI polymerized on S/SWNT to encapsulate sulfur and trap polysulfides, PANI-S/SWNT (c) and life cycle performance (d) of S/SWNT (black) and PANI-S/SWNT (red). PANI coated S/SWNT shows better capacity retention and coulombic efficiency after 100 cycles. Reprinted with permission from Kim JH, Fu K, Choi J, Kil K, Kim J, Han X, Hu L, Paik U: Encapsulation of S/SWNT with PANI web for enhanced rate and cycle performance in lithium sulfur batteries. Copyright 2015 Nature Publishing Group. 